\title{
Applying cognitive acuity theory to the development and scoring of situational judgment tests
}

\author{
J. Peter Leeds ${ }^{1}$
}

Published online: 9 November 2017

(C) Psychonomic Society, Inc. 2017

\begin{abstract}
The theory of cognitive acuity (TCA) treats the response options within items as signals to be detected and uses psychophysical methods to estimate the respondents' sensitivity to these signals. Such a framework offers new methods to construct and score situational judgment tests (SJT). Leeds (2012) defined cognitive acuity as the capacity to discern correctness and distinguish between correctness differences among simultaneously presented situationspecific response options. In this study, SJT response options were paired in order to offer the respondent a two-option choice. The contrast in correctness valence between the two options determined the magnitude of signal emission, with larger signals portending a higher probability of detection. A logarithmic relation was found between correctness valence contrast (signal stimulus) and its detectability (sensation response). Respondent sensitivity to such signals was measured and found to be related to the criterion variables. The linkage between psychophysics and elemental psychometrics may offer new directions for measurement theory.
\end{abstract}

Keywords Cognitive acuity · Situational judgment . Decision-making $\cdot$ Personnel assessment

In recent years, situational judgment tests (SJT) have become very popular in both research and applied personnel selection. However, little is understood about the underlying processes involved in how people

J. Peter Leeds

iamjleeds@gmail.com

1 University in Baltimore, 1420 N Charles Street, Baltimore, MD 21201, USA evaluate and select the response options on such tests. This article proposes examining SJT response options as psychophysical signals in order to both clarify these processes and to offer new methods for constructing and scorings such tests. Toward these ends, Leeds (2012) described a theory of cognitive acuity (TCA) that treats situational judgment test (SJT) response options as signals to be detected on the basis of their correctness valence and proposed that respondents' signal sensitivity could be measured to produce coefficients having direct and indirect criterion-related validity effects. Leeds defined cognitive acuity as the capacity to detect correctness and to distinguish between differences in correctness among simultaneously presented situation-specific response options. TCA departs from previous psychometric theories in that it proposes examining cognitive ability using psychophysical methods in a signal detection model of mental processing. The present article applies TCA in two SJT development and validation efforts - one for public service mobility reservation agents and the other for police officer candidates.

TCA proposes measuring properties within response options as the elemental level of psychometric observation. TCA seeks to estimate mean correctness levels of response options within test items by sampling subject matter expert (SMEs) and then presents response options to respondents as contrasted pairs. These pairings present the respondent with correctness contrast as a signal to be detected with detectability a function of (1) how much contrast there is and (2) how sensitive the respondent is to that contrast. It is this sensitivity that TCA seeks to measure using signal detection methods in order to estimate the respondent's construct possession. This methodology extends the boundaries of psychometrics to the most elemental level of the test - the response option itself. 

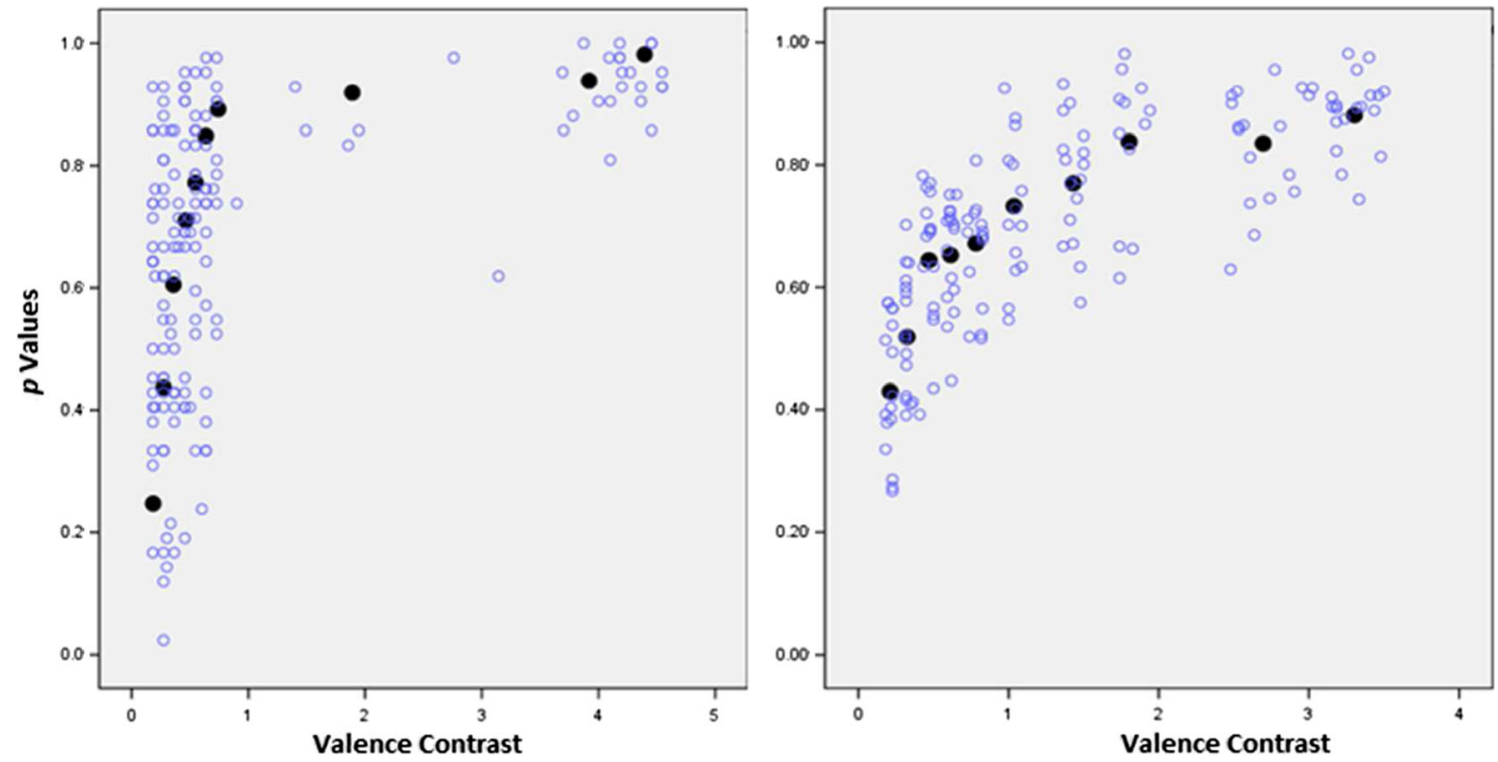

Graphs $1 \& 2$ Logarithmic relation between SJT item pair correctness valence contrast (signal stimulus) and $p$ value as the probability of correct detection (sensation response) in the two studies

\section{The mechanics of acuity theory measurement}

TCA focuses on the response option by recognizing that psychological constructs may be tapped not only at the total test score and item level, but also at the item response option level. TCA treats test item response options as if they were "emitting" construct-relevant signals and seeks to measure sensitivity to these signals using psychophysical methods. Just as an audiologist may administer varying sound signal strengths in order to gage sound acuity, the TCA method presents response options as correctness signals (e.g., in contrasted pairs) of varying strength $x$ and observes respondents' detection probabilities $\left(p\right.$ values $\left.^{1}\right)$ as sensitivity $y$. In this two-parameter model, the observed sensitivity of the respondent to SJT item response option correctness contrast (i.e., paired comparison) becomes the measure of signal sensitivity and hence degree of construct possession. Those respondents who are more sensitive to this signal are assumed to be more likely to possess and ultimately manifest the construct. TCA assumes that some response options emit stronger correctness signals than others and that some people are more sensitive to such signal (by virtue of their construct possession) than others. For example, a paired comparison SJT item for elementary school teachers may present the following stem:

You are writing an assignment on the board at the front of your 5th grade class. You turn your head to see one

\footnotetext{
${ }^{1}$ I will use the concept of $p$ value throughout this article, defined here as both (1) the probability of providing a correct answer and (2) the proportion of correct answers observed.
}

student push another. Which of the following is the better of the two response options?

Reprimand both for fighting.

Reprimand the one who pushed the other.

The more one possesses the situational judgment construct, the more sensitive one is to the signal emission, both in absolute terms (i.e., "how effective is this response option?") and in relative terms (i.e., "is option ' $A$ ' stronger or weaker construct-relevant signal than option 'B'?"). Assume that the construct being targeted is teacher conflict management and that the two response options emit different levels of correctness signal as estimated by SMEs. Suppose the SMEs estimated response option A as emitting a correctness signal of $(+2)$ (on $\mathrm{a}-3$ to +3 scale of correctness) and response option $\mathrm{B}$ a signal of $(-1)$ as it is an incorrect response. Each response option may be evaluated in absolute terms by presenting it in isolation and eliciting a "tends to be effective" $(+)$ or "tends to be ineffective" $(-)$ response from the respondent. Leeds (2012) showed that it is not the directionality (i.e., valence) of the correctness signal but rather its absolute magnitude that determines the likelihood of signal detection (i.e., getting the item right). Response options within items may also be evaluated in relative terms by their contrast to one another. For example, the correctness valence contrast between these two options is three units, or $|(-1)-(+2)|$. This level of contrast is easier to detect than it is to detect whether A or B "tends to be effective" individually. This is because three units of effectiveness contrast signal is easier to detect than either $|1|$ or $|2|$ units of effectiveness signal in isolation. In constructing SJTs, developers may wish to take these absolute and relative signals 
strengths into account when composing SJT items. The TCA research suggests that the SJT item's difficulty and hence its discrimination may be manipulated prior to administration with the effect of perhaps improving item parameters.

\section{The logarithmic relation between signal (correctness contrast) and sensation (observed $p$ value)}

As was reported by Leeds (2012), response option contrast and $p$ values are strongly related and this signal detection relationship conforms to a logarithmic function. SJT developers may capitalize on this known relationship between signal (contrast) and sensation ( $p$ value) to estimate respondents' sensitivity to correctness signals (i.e., cognitive acuity) and observe the degree to which this sensitivity is related to external criteria (e.g., construct relevant competencies). The work of Ernst Heinrich Weber (1795-1878) and Gustav Theodor Fechner (1801-1887) provide a framework for investigating stimulus-sensation relationships and the TCA. The WeberFechner law states that an organism's physiological or psychological response is a function of the logarithm of stimulus intensity. That is, stimulus detectability is logarithmically related to stimulus magnitude. This law has been observed across many forms of stimuli over many years of investigation with applications to light intensity (Hecht, 1924), loudness (Harris, 1963), neural responses (Nieder \& Miller, 2004), and even extends to other cognitive domains of psychology with Tzur, Berger, Luria, and Posner (2010) applying it to human brain theta rhythm in detecting incorrect arithmetic equations. To the extent that this law generalizes to SJT's, it is expected that the relation between response option signal strength (i.e., both in terms of absolute and relative valence) and the probability of getting an SJT item correct (i.e., $p$ value) should be linearly (or curvilinearly) related. Post administration inspection of such relations may indicate that some SJT response options are performing better than others, and hence provide an additional important piece of information not provided by traditional item analysis methods.

TCA methods can also be used to estimate for each respondent his/her sensitivity to the SJT response option correctness signal. These signals arise from selectively pairing SJT item response options that have been calibrated by SMEs to emit different degrees of signal strength. Specifically, each response option will have been rated by SMEs, on a numeric scale, as to its correctness level. The difference between the two paired response options serves as the correctness signal to be detected by the respondent. The closer the mean correctness ratings (i.e., smaller contrast) between two response options, the weaker the signal, the more difficult it is to detect, and thus the lower the expected $p$ value. The larger the contrast, the easier it is to detect the more correct response option and the higher the expected $p$ value. Response option pairs are presented to the respondent for him/her to choose the option with the more positive (or less negative) correctness valence. After the respondent choses one of the two response options, we observe a dichotomous variable indicating correct or incorrect detection of that signal (i.e., $p$ value) by the respondent. An SJT scenario may have multiple paired comparison response options attached to an SJT stem and hence present multiple opportunities for the respondent to demonstrate sensitivity to the contrast signals. TCA assumes that sensitivity to these signals is indicative of possession of the construct of interest, in this case, situational judgment.

\section{The use of binary logistic regression analysis to estimate each respondent's signal sensitivity}

Treating paired SJT item response options as if they emitted "correctness contrast" signal to be detected is an unorthodox method for measuring respondents' situational judgment and special analysis methods may be helpful in estimating this sensitivity. Although simply counting the number of correct detections (i.e., raw score) may produce valid SJT scores, Leeds (2012) and Leeds and White (2013) showed that estimated signal sensitivity acted as the initial variable in a mediated relation between sensitivity, SJT raw score, and job performance. This research suggests that SJT response option correctness sensitivity is an important part of the mechanism by which SJTs measure their construct. This research also suggests that SJT response option signal sensitivity is different from SJT item-level performance (e.g., raw scores) and should be measured separately. Specifically, if response option signal sensitivity impacts item-level performance-which in turn impacts external criteria (e.g., job performance), then careful attention should be paid to the signal transmissions of the response options and how the options are arranged in the item to present this signal. If SJT item response option signal sensitivity is important to SJT item performance and perhaps directly or indirectly to external criteria, then measuring this sensitivity may be informative. However, methods for measuring SJT item response option signal sensitivity have not been well established in the literature and requires special methods.

Acuity scoring of an SJT item requires taking into consideration the correctness valence of the response options both in terms of absolute valence (e.g. "how good is this option") and in terms of relative valence (e.g., how much better is this option than that option) as estimated by SMEs. When people respond to an SJT item, they select (or rate, depending on scoring scheme) one response option as compared to another. Since the correctness valence will have already been assigned to each response option by the SMEs, recording the person's correct or incorrect choice will produce a set of binary dependents (i.e., 0 or 1 ) and a set of continuous independents (the 
SME correctness valence). One solution to estimating the extent to which the valence (continuous variable) contributed to the person getting the response option correct $(0,1)$ is to use binary logistic regression analysis (BLRA). Specifically, the resulting within-person logistic beta weight will serve as a measure of the degree to which the person relied on the response option pair contrasts to discern the correctness of the competing response options in the pairs. Such a within-person analysis allows the researcher to estimate for each respondent the degree to which correct versus incorrect signal detections are a function of correctness valence contrast signal that was emitted and then detected. Those respondents who rely more strongly on this signal to make their binary choices are assumed to be more sensitive (or acute) to this signal than those who rely less strongly. As such, the beta weights derived from the BLRA may provide a measure of the strength of relationship between IV (signal) and DV (sensation) and thus estimate signal sensitivity.

To summarize, the logistic beta weights are regression coefficients that function as a persons' SJT score. These weights are obtained by regressing for each person (1) the column of SJT item response option pair correctness values, 0 or 1 , on (2) the column of SJT item response option pair contrast level (observed as the differences between each pair's SME-rated effectiveness contrast-i.e., signal strength). Thus, each person's SJT score is essentially weighted by the signal strength or difficulty of the response option pairs. By weighting items by the signal strength, higher SJT scores (i.e., larger regression coefficients) are obtained by those who are more sensitive to the signal and hence considered to be in greater possession of the situational judgment characteristic. This scoring system attempts to give deference to the correctness signal emissions of the response options, in effect weighting the resulting scores by the degree of obviousness of the response options.

\section{Statements of hypotheses}

Leeds (2012) originally used SJTs having eight response options per item stem with contrast defined as the difference between the mean of the four negatively valenced response options and mean of the four positively valenced response options (i.e., four effective and four ineffective options). I used a more parsimoneous method of developing SJT response options to capitalize on a paired comparison method in order to estimate respondent's correctness sensitivity (i.e., cognitive acuity) and more accurately measure the underlying construct. For this study I used a sample of reservation agents and police officer candidates.

Hypothesis 1 states that the BLRA-derived cognitive acuity estimates will be significantly related to both reservation agent job performance and to police officer candidate competency measures.
Leeds (2012) showed that respondent's binary responses to a multiple-choice SJT could be modeled as a logarithmic function of response option correctness level to response option correctness detection. Therefore, Hypothesis 2 states that the relationship between item response option pair contrast (signal stimulus) and probability of correct response option selection (sensation response) will be significantly logarithmic in both samples.

Beyond the goal of developing deeper understanding of how people make decisions when faced with difficult choices, TCA may provide an improvement to traditional raw binary scores (e.g., one point awarded for each correct answer and none for an incorrect answer). Although other scoring schemes exist (e.g. pick the most and the least effective, rank order response options), raw binary scoring is the basic test scoring method and serves as baseline to compare other methods. Therefore, Hypothesis 3 states that the BLRAderived cognitive acuity estimates will account for significant reservation agent job performance and police officer candidate competence variance above and beyond that accounted for by the SJT raw binary scores.

\section{Method}

\section{Participants}

Study 1 A group of 12 reservation agent SMEs from a large urban municipality were used in the SJT development. The SMEs reported extensive on-the-job experience, with an average of 15.7 years. In addition, 42 active reservation agents were recruited as job incumbent test-taking respondents for this study. All respondents were Black, with eight being male and 34 female. The participating incumbents were recruited for participation voluntarily as part of the agency's test development efforts and were on the clock during test administration.

Study 2 A group of 24 police officer subject matter experts from large municipal police departments were used in the SJT development. A total of 162 police officer candidates from a single large metropolitan police department were assessed on various measures as part of their preemployment screening. This number was reduced to 144 candidates after removing those who omitted more than 16 test items and or had a total test $p$ value $<.55$, indicating random responses. It should be noted that these police officer candidates were taking a rigorous battery of assessments and were informed that the experimental SJT would not be used in any way for selection decision-making. The candidates were administered the SJT and all performance criterion measures after graduating the police academy but before serving as police officers. Their racial subgroup membership included two Asians, five Hispanics, 
92 Whites, 35 Blacks, one Native American, and nine unidentified. The sample comprised 18 females and 121 males, with five unidentified.

\section{Acuity measures (Studies 1 and 2)}

An audio SJT was developed for Study 1 that presented a series of ten audio scenarios of scripted critical incidents extracted from actual reservation agent telephone calls to create a high-fidelity audio SJT. The video SJT developed for Study 2 used content-validated critical incidents that were scripted and filmed for the purpose of providing difficult scenarios to prospective police officers. For both studies two different sets of SMEs generated 20-40 response options for each scenario having an about equal proportion of effective, neutral, and ineffective responses. Each response was then rated by the study's respective SMEs on effectiveness. This effectiveness valence ranged from highly ineffective $(-3)$ to highly effective $(+3)$ with zero indicating neutrality. The mean SME rating identified the correctness valence contrast level of the response option. SMEs showing poor inter-rater agreement with the other SMEs were removed from the analyses. Response options were evaluated for SME consistency using Brown and Hauenstein (2005) coefficient of agreement $a_{W G(1)}$ (a variant of $\mathrm{r}_{W G}$ ). The $a_{W G(1)}$ uses the maximum possible variance in the denominator rather than the variance of the uniform distribution. Those response options producing $a_{W G(1)}<.80$ were removed from the analyses. This procedure was used to reduce the correctness signal distortion emitted by response options having equivocal signal strength. By removing such item pairs, I sought to improve the reliability of the correctness signal emission.

In both sets of data, surviving responses were paired to present a range of correctness valence contrast anticipated to be useful in measuring signal sensitivity across a wide range of signal strengths. The absolute correctness valence contrast between the two response options in an item was calculated as the signal strength of the item. In Study 1, a total of 156 paired comparison SJT items were developed and distributed across the ten audio scenarios. Audio scenarios were developed using dispatcher SMEs and job analysis based on the critical incident technique (Flanagan, 1954). Each scenario presented a different audio scenario that tapped different SME-content validated knowledge, skills, and abilities. Scenarios had different numbers of response option pairs and respondents were required to pick the best response from each pair. Scenario 1 had five pairs $(1,5)$, and Scenario 2 had 59 pairs $(2,59)$, with the remaining scenarios denoted as follows: $(3,13) ;(4,10)$; $(5,15) ;(6,9) ;(7,17) ;(8,16) ;(9,6)$; and $(10,6)$. Coefficient alpha for this SJT was .70. In Study 2, a total of seven video scenarios and 195 paired comparison items were developed for the video SJT. Scenario 1 had 31 response option pairs (1, $31)$, with the remaining scenarios denoted as follows: $(2,26)$;
$(3,14) ;(4,40) ;(5,36) ;(6,11)$; and $(7,37)$. The video scenarios were developed and content-validated using police SMEs and job analysis based on the critical-incident technique. Coefficient alpha for this SJT was .82.

In both studies, testing was proctored and respondents listened to or viewed the scenarios and responded on paper to the series of paired comparison items. Scores were generated from the two SJT administrations with both conventional and TCA scoring methods applied.

Conventional method SJT_Raw_Binary_Total scores are the sum of applicants' binary ( 1 point for a correct response, and 0 for an incorrect) responses to the items on the SJT.

TCA method Binary logistic regression analysis (BLRA) statistics were generated by fitting a logistic regression function to each respondent's set of binary responses (the dependent variable) and SME-estimated correctness valence contrasts (the independent variable). A separate logistic regression was conducted for each respondent and the regression parameters were obtained from the output. The results of this analysis method generated what we will label as the Logistic_Beta scores, which are the unstandardized logistic regression beta weights having the same interpretation as the weights from OLS regression. These scores will serve as estimates of the degree to which the respondent attended to the signal strength in deciding to select a response option.

\section{Dependent and criterion variables}

\section{Study 1 -Reservation agent study}

Establishing criterion variables in this study involved collecting performance appraisal ratings from the supervisors of the participating reservation agents. The appraisal measure instrument was developed by the author and a graduate student working in the facility and was designed, on the basis of job analysis, to tap the full domain of the job. Six performance ratings of job performance were collected from each participant's immediate supervisor. A 5-point scale was developed with rating scale values ranging from 1 (marginal) to 5 (excellent). These dimensions reflected the job domains presented in the SJT audio scenarios and include: PA $1=$ telephonically managing panicked clients; PA $2=$ managing confused clients over the phone; PA 3 = managing angry clients; PA $4=$ decision making effectiveness; PA $5=$ overall job performance; PA Total $=$ Sum PA 1 through PA 5. Rating PA1 to PA6 had a combined coefficient alpha $=.95$, establishing internal consistency reliability. 


\section{Study 2-Police officer candidate study}

In addition to the experimental SJT, the following instruments were administered to police officer candidates. Since no formal job performance information was available from these candidates, scores obtained from these instruments served as criterion-relevant dependent variables as they are measures of candidate competence. These instruments were used by the police academy as part of their screening and assessment program developed by professional psychologists for use in evaluating armed law enforcement officers. No formal test characteristics were made available to the author. The measures included:

Multiple-choice test A test of police training academy success potential focusing on the knowledge, skills, and abilities needed in the academy. The test includes vocabulary, spelling, reading comprehension, and map reading.

BPAD The Behavioral Personnel Assessment Device (BPAD) is a commercial high-fidelity video scenario simulator presenting candidates with difficult situations found in law enforcement. The candidates were videotaped responding to each of eight video scenarios as if they were experiencing the scenario in real time. A trained assessor rates the recorded responses on a $1-4$ scale of effectiveness. The BPAD is not a test of knowledge or cognitive ability but rather a test of the candidate's interpersonal competence in dealing with different types of people in different job-specific situations.

Writing sample A video is shown to candidates who take notes and later write a report based on the information in the video. The report is scored on accurate presentation of information, correct grammar, and punctuation.

Information analysis Part of the writing sample with derived scores based on the candidate's ability to draw conclusions and offer critical evaluations of information.

\section{Results}

\section{Item analysis}

For the SJT_Raw_Binary_Total in Study 1 and Study 2, coefficient alpha was estimated at .68 and .70 respectively. For the acuity measure (i.e., Logistic Beta) no conventional item analyses statistics were available to assess item response option pair functioning. However, a theoretical model exists such that signals emitted by contrasted response option pairs and $p$ values should be logarithmically related (Leeds, 2012; Leeds \& White, 2013). It was therefore possible to evaluate items (i.e., pairs) on the basis of their conformity to the logarithmic function. That is, after fitting a logarithmic function to all item response option pair contrasts and their associated $p$ values, the largest residuals (i.e., those pairs that fell farthest from the fitted function do not conform to the theoretical logarithmic relation between signal and detection) were candidates for removal.

The intent of this removal was to demonstrate that a logistic function fit the difficulty- $p$-value relation and that removing items that poorly fit this function appeared to improve rather than degrade the validity of the remaining items. This analysis treats SJT response options (i.e., applying TCA) as signals to be detected and suggests the use of a psychophysical model (i.e., the Weber-Fechner function) in evaluating the SJT post administration. Just as alpha-if-item deleted, item-total-correlation, and item variance estimates are used as criteria for item performance evaluation in classical item analysis procedures, TCA suggests the method of examining $p$ value to signal valence relation as another method of evaluating SJT item performance post-administration. One may argue that SJT response options that are far more obvious (emit much stronger correctness signal) or less obvious (emit far lower signal) than the SME-determined valence would suggest, are performing strangely and both they and their SJT item stems should be flagged as unusual and examined more closely.

Using this method, a total of 44 response option pairs (i.e., the SJT items) were chosen for removal from the acuity measures in both studies because this number could be reasonably removed while still preserving enough signals to estimate respondents' acuity. Note that this procedure does not attempt to delete items showing poor validity thus over fitting the resulting prediction equations. Rather, it attempts to increase item precision by capitalizing on the known logarithmic relation between stimulus (valence contrast signal) and sensation (observed $p$ value) to remove nonconforming or poor-fitting response option pairs. It was on this reduced set that all validity analyses were based.

Hypothesis 1 stated that all estimates of cognitive acuity would be significantly related to the reservation agent job performance and police officer candidate competency dimensions. Spearman rank order correlations were used in Study 1 because of the small sample size and because the data were nonnormal according to the Shapiro-Wilk test of normality. Table 1 presents the descriptive statistics and the Spearman correlations between acuity scores and the dependent variables for Study 1.

In contrast to SJT_Raw_Binary_Total scores, which evidenced no significant correlations to any of the performance criteria, the Logistic Beta scores were significantly $(p<.01)$ correlated with all six performance dimensions, with coefficients ranging between $r=.43$ (managing angry clients and decision making) to $r=.58$ (managing panicked clients). Thus, Hypothesis 1 was supported in the reservation agent Study 1. 
Table 1 Study 1 means, standard deviations, and Spearman correlation coefficients $(N=42)$

\begin{tabular}{|c|c|c|c|c|c|c|c|c|c|c|}
\hline & Mean & $S D$ & 1 & 2 & 3 & 4 & 5 & 6 & 7 & 8 \\
\hline 1. PA Total = Sum PA through PA5. & 15.07 & 4.65 & $(.95)$ & & & & & & & \\
\hline 2. PA 1 (managing panicked clients) & 3.07 & 1.05 & $.88^{* * *}$ & 1 & & & & & & \\
\hline 3. PA 2 (managing confused clients) & 3.02 & 1.14 & $.92^{* *}$ & $.83^{* *}$ & 1 & & & & & \\
\hline 4. PA 3 (managed angry clients) & 3.02 & 1.14 & $.92^{* *}$ & $.77^{* * *}$ & $.86^{\text {** }}$ & 1 & & & & \\
\hline 5. PA 4 (decision making) & 2.93 & 0.97 & $.85^{* * *}$ & $.71^{* * *}$ & $.72^{* *}$ & $.73^{* *}$ & 1 & & & \\
\hline 6. PA $5=($ overall job performance $)$ & 3.00 & 0.77 & $.84^{* *}$ & $.85^{* *}$ & $.83^{* * *}$ & $.81^{* * *}$ & $.79^{* *}$ & 1 & & \\
\hline 7. SJT_Raw_Binary_Total & 80.45 & 7.79 & .18 & .07 & .14 & .13 & .12 & .16 & $(.68)$ & \\
\hline 8. Logistic_Beta & 1.16 & 1 & $.53^{* * *}$ & $.58^{* *}$ & $.51^{* * *}$ & $.43^{* *}$ & $.43^{* *}$ & $.45^{* *}$ & .23 & 1 \\
\hline
\end{tabular}

${ }^{*}$ Correlation is significant at the .05 level (two-tailed). ${ }^{* *}$ Correlation is significant at the .01 level (two-tailed). Coefficient alpha appears in the diagonal when it was available or estimable. SJT_Raw_Binary_Total = the sum of applicants' binary (1 point for a correct response and 0 for an incorrect) responses to the items on the SJT. Logistic_Beta $=$ the unstandardized logistic regression beta weights for each respondent's fitted model

Hypothesis 1 was evaluated in Study 2 with results presented in Table 2, which shows the subgroup difference effect sizes, descriptive statistics, and correlations of the SJT scores and the dependent measures of police officer candidate competence.

Consistent with the finding of Leeds (2012), the cognitive acuity scores tended to be positively skewed and nonnormal, and thus Cliff's (1993) Delta was computed as a nonparametric equivalent to Cohen's $d$ for estimating the size of the Black-versus-White subgroup differences. The sample sizes precluded analysis of the other racial groups. Delta was estimated using software by Young (1994) and Ledesma, Macbeth, and Cortada de Kohan (2009). Where acuity measures were nonnormal, the Delta was used to estimate the effect size, with significant differences between racial groups being assessed with the Mann-Whitney $U$. Where the acuity estimates were normal, Cohen's $d$ was used and differences were assessed with a $t$ test. SJT_Raw_Binary_Total was normally distributed and had a small, nonsignificant subgroup effect, with $d=-0.07$. Logistic_Beta was nonnormal and evidenced significant subgroup effects (favoring Whites), with Delta $=-.38$. These may be considered medium-size effects (Cohen, 1988) and are consistent with the metaanalytic White-versus-Black subgroup differences found on SJTs, which were estimated at $d=0.38$ (Whetzel, McDaniel, \& Nguyen, 2008).

Returning to Hypothesis 1 in Study 2, the Logistic_Beta correlated significantly $(p<.01)$ with multiple-choice test, writing skill test, and the information analysis test measures, with $r \mathrm{~s}=.37, .26$, and .29 respectively. Thus, Hypothesis 1 was also supported in Study 2.

Hypothesis 2 stated that the relationship between the item response option pair correctness valence contrast (i.e., the signal stimulus) and the $p$ value (the probability of correct response selection; i.e., the sensation response) would be significantly logarithmic in both samples. The logarithmic and linear relations between $p$ and signal were modeled using the baseline (prior to logarithmic outlier reduction) data from both studies. Graphs 1 and 2 present the overlaid scatterplots for the Study 1 and Study 2 results. The analyses were based on 156 SJT items from Study 1 and 195 SJT items in Study 2. These graphs show the logarithmic relation between $p$ values and

Table 2 Study 2: Black versus white subgroup difference effect sizes, means, standard deviations and Pearson correlation coefficients $(N=144)$

\begin{tabular}{llllllllll}
\hline & Cohen's $d$ & Cliff's Delta & Mean & SD & 1 & 2 & 3 & 4 & 5 \\
\hline 1. Multiple choice & $-0.45^{\dagger}$ & $-.25^{\dagger}$ & 65.92 & 9.53 & 1 & & & & \\
2. BPAD & -0.11 & -.16 & 61.03 & 12.96 & $.36^{* *}$ & 1 & & \\
3. Writing skill test & $0.84^{\dagger}$ & $.43^{\dagger}$ & 8.44 & 1.51 & $.49^{* *}$ & .19 & 1 & $.65^{* *}$ & 1 \\
4. Information analysis & $-0.45^{\dagger}$ & $-.28^{\dagger}$ & 7.9 & 1.7 & $.32^{* *}$ & .18 & $.65^{*}$ & $.21^{*}$ & $(.70)$ \\
5. SJT_Raw_Binary_Total & -0.07 & -.05 & 131.06 & 11.17 & .16 & .13 & $.22^{*}$ \\
6. Logistic_Beta & $-0.54^{\dagger}$ & $-.38^{\dagger}$ & .88 & .47 & $.37^{* *}$ & .16 & $.26^{* *}$ & $.29^{* *}$ & $.37^{* *}$ \\
\hline
\end{tabular}

${ }^{*}$ Correlation is significant at the .05 level (two-tailed). ${ }^{* *}$ Correlation is significant at the .01 level (two-tailed). ${ }^{\dagger}$ Subgroup differences favored Whites over Blacks and were significant $(p<.05)$ using either the $T$ test for Cohen's $d$ or the Mann-Whitney $U$ (two-tailed) for Cliffs Delta. * The data were significantly $(p<.05)$ nonnormal according to Shapiro-Wilk test of normality and thus Cliff's Delta is the preferred measure of subgroup differences. Coefficient alpha appears in the diagonal when it was available or estimable. SJT_Raw_Binary_Total = the sum of applicants' binary $(1$ point for a correct response and 0 for an incorrect) responses to the items on the SJT. Logistic Beta $=$ the unstandardized logistic regression beta weights for each respondent's fitted model 
correctness valence contrast, both in raw form (circles) and as means (solid dots), formed by aggregating ten homogeneous sets of $p$ values and ten homogeneous sets of correctness valence contrast. Taking the mean of the homogeneous sets was done to summarize the widely varying $p$ value and correctness valence contrasts developed in these studies.

In Study 1, the average $p$ values and their associated average correctness valence contrasts were computed for ten correctness valence contrast (signal) magnitudes, ranging from 0.18 to 4.5 . At each of the ten magnitudes, between five and 20 relatively similar examples of each magnitude were available to form the means (solid dots). In Study 2 there were ten magnitudes, ranging from 0.21 to 3.3. At each of these ten magnitudes, between ten and 25 relatively similar examples of each magnitude were available to form the means (solid dots).

In Study 1, the linear coefficient of determination for the raw data was $R^{2}=.25(p<.05)$, and the logarithmic value for the raw data was $R^{2}=.28(p<.05)$. For the mean data (homogeneous sets), a linear $R^{2}=.43(p<.05)$ was observed, whereas a logarithmic function produced an $R^{2}=.73(p<.05)$. In Study 2, the linear coefficient for the raw data was $R^{2}=.53$ $(p<.05)$, whereas the logarithmic value for the raw data was $R^{2}=.64(p<.05)$. For the mean data (homogeneous sets), a linear coefficient of determination of $R^{2}=.76(p<.05)$ was observed, whereas a logarithmic function produced an $R^{2}=$ $.96(p<.05)$. Thus, consistent with the findings of Leeds (2012), the data appear to suggest more of a logarithmic than a linear relation between valance contrast (signal) and the probability of detection (sensation), and this conclusion held whether the data analyzed were raw or based on the means of the homogeneous $p$ value and correctness valence sets. Hypothesis 2 was supported.

Hypothesis 3 stated that the BLRA-equation-derived cognitive acuity scores would account for significant dependentvariable (agent job performance and police officer candidate competence) variance above and beyond that accounted for by the SJT_Raw_Binary_Total scoring method. In Study 1, where none of the SJT_Raw_Binary_Total score criterion correlations were significant, the cognitive acuity estimates were significantly correlated to each of the six performance ratings. These significant correlations are presented in Table 1. The Logistic_Beta scores significantly $(p<.01)$ correlated with the all six criterions variables, with relations ranging between $r=$ .43 , for PA 3 (managing angry clients) and PA 4 (decisionmaking), to $r=.58$, for PA 1 (managing panicked clients). Thus, in Study 1 Hypothesis 3 was supported.

To test Hypothesis 3 in Study 2, separate hierarchical regression analyses were conducted treating SJT_Raw_Binary_Total as the control entered in Step 1, and the acuity estimates as the independent variable were entered at Step 2. However, because SJT_Raw_Binary_Total failed to significantly correlate to either the multiple-choice test or the BPAD, analyses will be limited to the writing skill and information analysis dependent measures, for which the SJT_Raw_Binary_Total evidenced a significant relation. Table 3 presents the results of these hierarchical regression analyses.

The results show that in separate hierarchical regression analyses, the independent variables contributed significantly $(p<.05)$ to the prediction of both writing skills and information analysis, above and beyond the portions accounted for by SJT_Raw_Binary_Total. For the writing skill dependent variable, significant $(p<.05)$ improvements to $R^{2}$ were observed for the independent variable of Logisit_Regression_Beta $\left(\Delta R^{2}=.04\right)$. For the information analysis dependent variable, significant $(p<.05) \mathrm{im}$ provements to $R^{2}$ were again observed for the Logisit_Regression_Beta $\left(\Delta R^{2}=.06\right)$.

Though the SJT_Raw_Binary_Total was found to be significantly correlated to writing skill and information analysis, the hierarchical regression presented in Table 3 indicates that significant additional criterion variance was also accounted for by the acuity measure. Thus, in both studies, the results generally indicated that the BLRA-derived cognitive acuity estimates accounted for dependent variation (reservation agent job performance and police officer competence) above that accounted for by traditional binary scoring (i.e., SJT_Raw_Binary_Total). Thus, Hypothesis 3 was generally supported with the BPAD results non-significent for neither TCA nor conventional scoring methods.

Table 3 Study 2 summary of hierarchical regression analyses of cognitive acuity scores predicting police officer writing skill and information analysis $(N=144)$

\begin{tabular}{|c|c|c|c|c|c|c|c|}
\hline Model & $R$ & $R$ Square & Std. Error of the Estimate & $\Delta R$ Square & $\Delta F$ & $d f 2$ & Sig. $F$ Change \\
\hline \multicolumn{8}{|l|}{ Dependent Variable: Writing Skill Test } \\
\hline a. SJT_Raw_Binary_Total & .22 & .05 & 1.48 & .05 & 5.24 & 107 & 0.02 \\
\hline b.SJT_Raw_Binary_Total, Logisit_Beta & .29 & .09 & 1.46 & .04 & 4.54 & 106 & 0.04 \\
\hline \multicolumn{8}{|l|}{ Dependent Variable: Information Analysis } \\
\hline a. SJT_Raw_Binary_Total & .21 & .04 & 1.67 & .04 & 4.77 & 107 & 0.03 \\
\hline b.SJT_Raw_Binary_Total, Logisit_Beta & .31 & .10 & 1.63 & .06 & 6.55 & 106 & 0.01 \\
\hline
\end{tabular}




\section{Additional analyses}

Since TCA is based on a signal detection framework with psychophysical underpinnings, it seems reasonable that removing logarithmic outliers might reduce error and therefore increases the validity coefficients observed in both studies. Although not a stated hypothesis, this possibility warranted an exploratory set of moderated regressions. To pursue this idea, a logarithmic curve-fitting procedure was used to model the relation between each response option pair contrast (i.e., signal) and its $p$ values in both studies. Then, the 44 SJT item response option pairs having the highest absolute residual variance from the logistic function were removed from both samples. The Logisit_Beta acuity estimates were then recomputed on the basis of this reduced set of pairs. Next, correlations between these new variables and their respective dependent variables (e.g., PA ratings in Study 1 and the competence-relevant variables in Study 2) were computed in both studies. Finally, these new post-outlier-reduced validity coefficients were compared to the original baseline validity coefficients.

Table 4 presents these results for both studies, where the baseline coefficients are contiguously compared to the postoutlier-reduction coefficients. The results show that the removal of the 44 logarithmic outliers at least nominally increased the validity coefficients (Spearman's rho) in both studies. In Study 1, we see the SJT_Raw_Binary_Total score moving from $r=-.18$ to .23 , and Logistic_Beta moving from $r=$ .44 to .53 . These removals did not dramatically affect internal consistency reliability, with alpha moving from .70 under baseline to .68 after reduction. For Study 2, we see the validity coefficients increasing marginally for Logistic_Beta and each of the criterion scores. These coefficients remained stable for the SJT_Raw_Binary_Total scores against each of the criterion variables, despite the item removal. In Study 2 the removals resulted in a reduction in coefficient alpha from .80 to .70 . These results support the removal of paired- comparison SJT test items on the basis of their nonconformity to the expected logarithmic relation between observed $p$ values and the correctness valence contrasts. It is possible that the $p$ to-contrast logarithmic relation for each response option pair contrast might provide an additional piece of information to researchers wishing to examine postadministration item functioning, while offering an opportunity to reduce items without suffering decrements to validity.

\section{Discussion}

As an extension of Leeds (2012), this research presented two studies that applied the theory of cognitive acuity (TCA) to the development and construct and criterion-related validation of an SJT for reservation agents (Study 1) and police officer candidates (Study 2). Using SME estimations of response option correctness valence contrast, SJT item pairs were built to offer various levels of correctness signal strength contrast to respondents. In both studies, these paired comparison SJT items were developed in order to estimate respondents' sensitivity to contrast signals and use these estimates to predict important job-related competencies.

Individual respondents' SJT item pair $p$ values (correct detection proportions) were collected and matched to SJT item response option pair contrast signals. Consistent with the psychophysical literature, findings suggest that the relation between signal strength (correctness valence contrast) and signal detectability ( $p$ values) followed a logarithmic function with very high coefficients of determination. Thus, sensitivity to SJT response option correctness signals follows the same pattern as that found in other psychophysical phenomena including sound, touch, light, and taste.

The results of these two studies support the assertion that people's critical event management decision making ability may be predicated on their sensitivity to the correctness signal presented by each response option pair and how contrasted these options are to each other in terms of resolution

Table 4 Comparison of baseline validity coefficients and those same coefficients after 44 logarithmic outliers were removed

\begin{tabular}{|c|c|c|c|c|c|c|c|c|c|c|}
\hline & \multirow{2}{*}{\multicolumn{2}{|c|}{$\frac{\text { Study } 1(n=42)}{\text { DV: Total PA }}$}} & \multicolumn{8}{|c|}{ Study 2 ( $n=144)$} \\
\hline & & & \multicolumn{2}{|c|}{$\begin{array}{l}\text { DV: Multiple Choice } \\
\text { Test }\end{array}$} & \multicolumn{2}{|c|}{$\begin{array}{l}\text { DV: Information } \\
\text { Analysis }\end{array}$} & \multicolumn{2}{|c|}{ DV: Writing Skill Test } & \multicolumn{2}{|c|}{ DV: BPAD } \\
\hline & Baseline & $\begin{array}{l}\text { Post Outlier } \\
\text { Reduction }\end{array}$ & Baseline & $\begin{array}{l}\text { Post Outlier } \\
\text { Reduction }\end{array}$ & Baseline & $\begin{array}{l}\text { Post Outlier } \\
\text { Reduction }\end{array}$ & Baseline & $\begin{array}{l}\text { Post Outlier } \\
\text { Reduction }\end{array}$ & Baseline & $\begin{array}{l}\text { Post Outlier } \\
\text { Reduction }\end{array}$ \\
\hline $\begin{array}{l}\text { SJT_Raw___ } \\
\text { Binary Tot }\end{array}$ & -.18 & .23 & .16 & .16 & $.21^{*}$ & $.21^{*}$ & $.22^{*}$ & $.22^{*}$ & .13 & .13 \\
\hline Logistic_Beta & $.44^{* *}$ & $.53^{* *}$ & $.28^{* *}$ & $.37^{* * *}$ & $.24^{*}$ & $.29^{* *}$ & $.21^{*}$ & $.26^{* *}$ & .06 & .16 \\
\hline
\end{tabular}

Study 1 used Spearman's rho correlations, whereas Study 2 uses Pearson correlations. * Correlation is significant at the .05 level (two-tailed)

** Correlation is significant at the .01 level (two-tailed). Total PA $=$ total performance appraisal rating. SJT_Raw_Binary_Total = the sum of applicants' correct response to the binary items on the SJT. Logistic_Beta $=$ the unstandardized logistic regression beta weights for each respondent's fitted model 
"goodness" or correctness. The results are consistent with those of Leeds (2012) and Leeds and White (2013) showing that this sensitivity is related to SJT performance and to important performance competencies. As such, response option correctness signal may be shown to be an elemental psychometric unit of analysis since it appears to be a consistent direct indicator of SJT performance and a direct or indirect indicator of the behavior that th SJTe was intended to measure.

TCA uses psychophysical methods to estimate response-option-level signal emission and people's sensitivity to these signals with the goal of refining construct measurement. Findings here and in previous research indicate that focusing beyond the test item and on to the item response options may offer utility to SJT development researchers. Furthermore, TCA proposes new methods to measure latent constructs. The data presented here suggest that more careful attention be paid to the construct-relevant signals emitted by the responses options that comprise the items and offers some methodology for measuring and improving SJT item functioning.

The methods outlined may provide new directions for item development by focusing on the psychophysical characteristics of the response option rather than the test or the item. In both studies, finding suggest that SJT response option pair contrasts having better logarithmic relation to $p$ values, tend to produce equal or at least nominally stronger validity coefficients with fewer items (i.e., response option pairs). This supports the utility of removing items that show poor logarithmic fit. When this removal was performed sequentially (i.e., with the most outlying items removed one at a time) validity coefficients increased across the removal progression up to a point of inflexion after which removals produced reductions in validity. This finding suggests that as the test converges on a set of items conforming increasingly to the theoretical logarithmic relation between $p$ value and item response option-pair contrast (i.e., signal), the accuracy with which the construct is measured increases accordingly. In addition to pointing to new directions for measurement, this finding may provide researchers with another tool for identifying and removing poorly performing test items and thereby increasing test precision and validity.

The psychometric methods proposed by TCA may also be used to calibrate SJT items by requiring SME agreement as to response option correctness level and removing response options showing poor agreement and hence ambiguous correctness signal. Furthermore, considering the observed relationships found between correctness valence contrast and $p$ value $(r=.41$ in Study 1 and $r=.57$ in Study 2), researchers may estimate, a priori, the difficulty levels of paired-comparison SJT items, and thereby configure items and tests to increase variability and avoid items that are too easy or too difficult. An additional advantage to TCA methods is that for every response option (n) calibrated, $n(n-1) / 2$ paired comparison items are available for administration. Thus, six response options may be paired to produce 15 unique items of varying signal strength difficulty. This has utility for item development since options may be reused to form unique combinations of paired contrast within the same item.

One area for further exploration is the possibility of direct estimation of TCA parameters. Specifically, by jointly estimating (1) the latent response option correctness valence signal, (2) external criteria, and (3) noise levels (observed as SME variability in their valence estimates) increased precision and model parsimony may be archived. Such a direct estimation would avoid the reliance on the SME-estimated "ground truth," and may improve the relation between sensitivity estimates and the criterion variables of interest. Such modeling would more fully capitalize on all the available information and avoid the need to remove SMEs whose correctness valence ratings were inconsistent with the other SMEs (i.e., noise). Simultaneous estimates of respondents' sensitivity and response option variability may improve interpretation of the results and obviate the need to independently estimate beta coefficients and residuals on the line of best fit. This direct joint modeling would be similar to modern Thurstonian methods such as those presented by Selker, Lee, and Iyer (2017) and also Lee, Steyvers, and Miller (2014), albeit that these authors required rank order rather than the point estimates of the response option valences collected in the present studies.

The linkage between psychophysics and elemental psychometrics is intriguing and may offer new directions for measurement theory as we explore the basic units of psychometric relevance - response options within items. The findings here suggest that the construct signal emitted by test item response options offers valid information about a respondent's standing on the construct of interest and may hold promise for future research.

\section{References}

Brown, R. D., \& Hauenstein, N. M. A. (2005). Interrater agreement reconsidered: An alternative to the rwg indices. Organizational Research Methods, 8, 165-184.

Cliff, N. (1993). Dominance statistics: Ordinal analyses to answer ordinal questions. Psychological Bulletin, 114, 494-509. https://doi.org/10. 1037/0033-2909.114.3.494

Cohen, J. (1988). Statistical power analysis for the behavioral sciences (2nd ed.). Mahwah: Erlbaum.

Flanagan, J. C. (1954). The critical incident technique. Psychological Bulletin, 51, 327-358.

Harris, J. D. (1963). Loudness discrimination. Journal of Speech and Hearing Disorders (Monograph Supplement No. 11), 1-63.

Hecht, S. (1924). The visual discrimination of intensity and the WeberFechner Law. Journal of General Physiology, 7, 235-267. 
Ledesma, R. D., Macbeth, G., \& Cortada de Kohan, N. (2009). Computing effect size measures with ViSta-The Visual Statistics System. Tutorial in Quantitative Methods for Psychology, 5, 25-34.

Lee, M. D., Steyvers, M., \& Miller, B. J. (2014). A cognitive model for aggregating people's rankings. PLoS ONE, 9, e0096431. https://doi. org/10.1371/journal.pone.0096431

Leeds, J. P. (2012). The theory of cognitive acuity: Extending psychophysics to the measurement of situational judgment. Journal of Neuroscience, Psychology, and Economics, 5, 106-181.

Leeds, J. P., \& White, A. (2013). Estimating cognitive acuity in two situational judgment tests: More lessons from psychophysics. Paper presented at the 25th Annual Convention of the Association for Psychological Science, Washington, DC.

Nieder, A., \& Miller, E. K. (2004). Analog numerical representations in rhesus monkeys: Evidence for parallel processing. Journal of Cognitive Neuroscience, 16, 889-901.
Selker, R., Lee, M. D., \& Iyer, R. (2017). Thurstonian cognitive models for aggregating top- $n$ lists. Decision, 4, 87-101. https://doi.org/10. 1037/dec0000056

Sweet, S. A., \& Grace-Martin, K. (2003). Data analysis with SPSS: A first course in applied statistics (2nd ed.). Boston: Allyn \& Bacon.

Tzur, G., Berger, A., Luria, R., \& Posner, M. I. (2010). Theta synchrony supports Weber-Fechner and Stevens' Laws for error processing, uniting high and low mental processes. Psychophysiology, 47, 758-766. https://doi.org/10.1111/j.1469-8986.2010.00967.x

Whetzel, D. L., McDaniel, M. A., \& Nguyen, N. T. (2008). Subgroup differences in situational judgment test performance: A meta-analysis. Human Performance, 21, 291-309. https://doi.org/10.1080/ 08959280802137820

Young, F. W. (1994). ViSta: The Visual Statistics System (Version 6.4) [computer software]. Available from www.visualstats.org 